\title{
Infinitely Many Homoclinic Orbits for 2nth-Order Nonlinear Functional Difference Equations Involving the $p$-Laplacian
}

\author{
Xiaofei He $\mathbf{H e}^{1,2}$ \\ ${ }^{1}$ Department of Mathematics and Computer Science, Jishou University, Hunan, Jishou 416000, China \\ ${ }^{2}$ Zhangjiajie College of Jishou University, Zhangjiajie 427000, China
}

Correspondence should be addressed to Xiaofei He, hxfcsu@sina.com

Received 14 October 2011; Accepted 18 November 2011

Academic Editor: Donal O’Regan

Copyright (c) 2012 Xiaofei He. This is an open access article distributed under the Creative Commons Attribution License, which permits unrestricted use, distribution, and reproduction in any medium, provided the original work is properly cited.

By establishing a new proper variational framework and using the critical point theory, we establish some new existence criteria to guarantee that the $2 n$ th-order nonlinear difference equation containing both advance and retardation with $p$-Laplacian $\Delta^{n}\left(r(t-n) \varphi_{p}\left(\Delta^{n} u(t-1)\right)\right)+$ $q(t) \varphi_{p}(u(t))=f(t, u(t+n), \ldots, u(t), \ldots, u(t-n)), n \in \mathbb{Z}(3), t \in \mathbb{Z}$, has infinitely many homoclinic orbits, where $\varphi_{p}(s)$ is $p$-Laplacian operator; $\varphi_{p}(s)=|s|^{p-2} s(1<p<\infty) r, q, f$ are nonperiodic in $t$. Our conditions on the potential are rather relaxed, and some existing results in the literature are improved.

\section{Introduction}

In this paper, we shall be concerned with the existence of homoclinic orbits of the nonlinear difference equation

$$
\begin{aligned}
& \Delta^{n}\left(r(t-n) \varphi_{p}\left(\Delta^{n} u(t-1)\right)\right)+q(t) \varphi_{p}(u(t)) \\
& \quad=f(t, u(t+n), \ldots, u(t), \ldots, u(t-n)), \quad n \in \mathbb{Z}(3), t \in \mathbb{Z},
\end{aligned}
$$

where $\Delta$ is the forward difference operator defined by $\Delta u(t)=u(t+1)-u(t), \Delta^{2} u(t)=$ $\Delta(\Delta u(t)) \cdot \varphi_{p}(s)$ is $p$-Laplacian operator $\varphi_{p}(s)=|s|^{p-2} s(1<p<\infty)$. As usual, we say that a solution $u(t)$ of (1.1) is homoclinic (to 0 ) if $u(t) \rightarrow 0$ as $t \rightarrow \pm \infty$. In addition, if $u(t) \not \equiv 0$, then $u(t)$ is called a nontrivial homoclinic solution. It is well known that homoclinic orbits play an 
important role in analyzing the chaos of dynamical systems; we refer the interested reader to [1-15].

We may think of (1.1) as being a discrete analogue of the $2 n$ th-order differential equation

$$
\left[r(t) \varphi_{p}\left(x^{(n)}\right)\right]^{(n)}+q(t) \varphi_{p}(x)-f(t, x(t+n), \ldots, x(t), \ldots, x(t-n))=0, \quad t \in \mathbb{R} .
$$

If $p=2,(1.1)$ reduces to the following equation:

$$
\Delta^{n}\left(r(t-n) \Delta^{n} u(t-n)\right)+q(t) u(t)=f(t, u(t+n), \ldots, u(t), \ldots, u(t-n)), \quad n \in \mathbb{Z}(3), \quad t \in \mathbb{Z}
$$

Recently, Chen and Tang [2] applied the critical point theory to prove the existence of homoclinic solutions for (1.3).

In some recent papers $[1,2,8,9,16-18]$, the authors studied the existence of periodic and homoclinic solutions of second-order nonlinear difference equation by using the critical point theory. These papers show that the critical point method is an effective approach to the study of periodic solutions of second-order difference equations. Compared to oneorder or second-order difference equations, the study of higher-order equations has received considerably less attention (see, e.g., $[2,3,19-21]$ and references contained therein). But to the best knowledge of the authors, results on existence of homoclinic solutions of (1.1) have not been found in the literature.

Motivated by the recent papers $[2,5,14,22]$, the aim of this paper is to consider problem (1.1) in a more general sense. More exactly our results represent the extensions to equations with $p$-Laplacian. Throughout the paper, for a function $G$, we let $G_{i}^{\prime}\left(x_{1}, x_{2}, \ldots, x_{i}, \ldots\right.$, $x_{n}$ ) denote the partial derivative of $G$ on the $i$ variable.

\section{Main Results}

Theorem 2.1. Assume that $q$ and $F$ satisfy the following assumptions:

(r) For every $t \in \mathbb{Z}, r(t)>0$;

(q) For every $t \in \mathbb{Z}, q(t)>0$, and $\lim _{|t| \rightarrow+\infty} q(t)=+\infty$;

(F1) There exists a functional $F\left(t, x_{n}(t), \ldots, x_{0}(t)\right)$ which is continuously differentiable in the variable from $x_{n}$ to $x_{0}$ for every $t \in \mathbb{Z}$ and satisfy

$$
\begin{gathered}
\sum_{i=-n}^{0} F_{2+n+i}^{\prime}\left(t+i, x_{n+i}, \ldots, x_{i}\right)=f\left(t, x_{n}, x_{n-1}, \ldots, x_{0}, x_{-1}, \ldots, x_{-n}\right), \\
\left|f\left(t, x_{n}, x_{n-1}, \ldots, x_{0}, x_{-1}, \ldots, x_{-n}\right)\right|=o\left(\sum_{i=-n}^{n}\left|x_{i}\right|^{p-1}\right), \quad \text { as } \sum_{i=-n}^{n}\left|x_{i}\right|^{p-1} \longrightarrow 0, \\
\left|F\left(t, x_{n}, \ldots, x_{0}\right)\right|=o\left(\sum_{i=-n}^{n}\left|x_{i}\right|^{p}\right), \quad \text { as } \sum_{i=-n}^{n}\left|x_{i}\right|^{p} \longrightarrow 0
\end{gathered}
$$

uniformly in $t \in \mathbb{Z} \backslash J$, where $J$ is defined in (F2); 
(F2) $F\left(t, x_{n}, \ldots, x_{0}\right)=W\left(t, x_{0}\right)-H\left(t, x_{n}, \ldots, x_{0}\right)$, for every $t \in \mathbb{Z}, W, H$ are continuously differentiable in $x_{0}$ and $x_{n}, \ldots, x_{0}$, respectively. Moreover, there is a bounded set $J \subset \mathbb{Z}$ such that

$$
H\left(t, x_{n}, \ldots, x_{0}\right) \geq 0
$$

(F3) There is a constant $\mu>p$ such that

$$
0<\mu W\left(t, x_{0}\right) \leq W_{2}^{\prime}\left(t, x_{0}\right) x_{0}, \quad \forall\left(t, x_{0}\right) \in \mathbb{Z} \times(\mathbb{R} \backslash\{0\})
$$

(F4) $H(t, 0, \ldots, 0) \equiv 0$, and there is a constant $\varrho \in(p, \mu)$ such that

$$
\sum_{i=-n}^{0} H_{2+n+i}^{\prime}\left(t, x_{n}, \ldots, x_{0}\right) x_{-i} \leq \rho H\left(t, x_{n}, \ldots, x_{0}\right)
$$

(F5) There exists a constant $b>0$ such that

$$
H\left(t, x_{n}, \ldots, x_{0}\right) \leq b \gamma^{\varrho}, \quad \text { for } t \in \mathbb{Z}, \gamma>1,
$$

where $\gamma=\left(\sum_{i=0}^{n}\left|x_{i}\right|^{p}\right)^{1 / p}$.

(F6) $F\left(t,-x_{n}, \ldots,-x_{0}\right)=F\left(t, x_{n}, \ldots, x_{0}\right)$, for all $\left(t, x_{n}, \ldots, x_{0}\right) \in \mathbb{Z} \times \mathbb{R}^{n+1}$.

Then (1.1) possesses an unbounded sequence of homoclinic solutions.

Theorem 2.2. Assume that $r, q$, and $F$ satisfy (r), (q), (F1), (F3)-(F5) and the following assumption:

(F2') $F\left(t, x_{n}, \ldots, x_{0}\right)=W\left(t, x_{0}\right)-H\left(t, x_{n}, \ldots, x_{0}\right)$, for every $t \in \mathbb{Z}, W, H$ are continuously differentiable in $x_{0}$ and $x_{n}, \ldots, x_{0}$, respectively, and

$$
\left|F\left(t, x_{n}, \ldots, x_{0}\right)\right|=o\left(\gamma^{p}\right) \text { as } \gamma \longrightarrow 0
$$

where $\gamma=\left(\sum_{i=0}^{n}\left|x_{i}\right|^{p}\right)^{1 / p}$ uniformly in $t \in \mathbb{Z}$.

Then (1.1) possesses an unbounded sequence of homoclinic solutions.

Theorem 2.3. Assume that $r, q$, and $F$ satisfy $(r),(q),(F 1)$ and satisfy the following assumptions:

(F7) For any $t \in \mathbb{Z}$,

$$
F\left(t, x_{n}, \ldots, x_{0}\right) \geq F\left(t, x_{0}\right) \geq 0
$$


(F8) For any $r>0$, there exist $a=a(r), b=b(r)>0$, and $v<p$ such that

$$
\begin{aligned}
& \left(p+\frac{1}{a+b\left(\sum_{i=0}^{n}\left|x_{i}\right|^{p}\right)^{v / p}}\right) F\left(t, x_{n}, \ldots, x_{0}\right) \\
& \quad \leq \sum_{i=-n}^{0} F_{2+n+i}^{\prime}\left(t, x_{n}, \ldots, x_{0}\right) x_{-i}, \quad \forall t \in \mathbb{Z},\left(\sum_{i=0}^{n}\left|x_{i}\right|^{p}\right)^{1 / p} \geq r ;
\end{aligned}
$$

(F9) For any $t \in \mathbb{Z}$

$$
\lim _{s \rightarrow+\infty}\left[s^{-p} \min _{|x|=1} F(t, s x)\right]=+\infty
$$

Then there exists an unbounded sequence of homoclinic solutions for (1.1).

\section{Preliminaries}

To apply critical point theory to study the existence of homoclinic solutions of (1.1), we shall state some basic notations and lemmas, which will be used in the proofs of our main results. Let

$$
\begin{gathered}
S=\left\{\{u(t)\}_{t \in \mathbb{Z}}: u(t) \in \mathbb{R}, t \in \mathbb{Z}\right\}, \\
E=\left\{u \in S: \sum_{t \in \mathbb{Z}}\left[\left|r(t-1) \Delta^{n} u(t-1)\right|^{p}+q(t)|u(t)|^{p}\right]<+\infty\right\}
\end{gathered}
$$

and for $u \in E$, let

$$
\|u\|=\left\{\sum_{t \in \mathbb{Z}}\left[\left|r(t-1) \Delta^{n} u(t-1)\right|^{p}+q(t)|u(t)|^{p}\right]\right\}^{1 / p} .
$$

Then $E$ is a uniform convex Banach space with this norm.

Let $I: E \rightarrow \mathbb{R}$ be defined by

$$
I(u)=\frac{1}{p}\|u\|^{p}-\sum_{t \in \mathbb{Z}} F(t, u(t+n), \ldots, u(t)) .
$$

If ( $q)$ and $(F 1)$ hold, then $I \in C^{1}(E, \mathbb{R})$ and one can easily check that

$$
\begin{aligned}
\left\langle I^{\prime}(u), v\right\rangle=\sum_{t \in \mathbb{Z}}[ & r(t-1)\left|\Delta^{n} u(t-1)\right|^{p-2} \Delta^{n} v(t-1)+q(t)|u(t)|^{p-2} v(t) \\
& -f(t), u(t+n), \ldots \mathrm{u}(t), \ldots u(t-n) v(t)], \quad \forall u, v \in E .
\end{aligned}
$$


By using

$$
\Delta^{n} u(t-1)=\sum_{k=0}^{n}(-1)^{k}\left(\begin{array}{l}
n \\
k
\end{array}\right) u(t+n-k-1)
$$

we can compute the partial derivative as

$$
\frac{\partial I(u)}{\partial u(t)}=\Delta^{n}\left(r(t-n) \varphi_{p}\left(\Delta^{n} u(t-1)\right)\right)+q(t) \varphi_{p}(u(t))-f(t, u(t+n), \ldots, u(t), \ldots, u(t-n))
$$

So, the critical points of $I$ in $E$ are the solutions of (1.1) with $u( \pm \infty)=0$.

Lemma 3.1 (see [12]). Let $E$ be a real Banach space and $I \in C^{1}(E, \mathbb{R})$ satisfy (PS)-condition with $I$ even. Suppose that I satisfies the following conditions:

(i) $I(0)=0$;

(ii) There exist constants $\rho, \alpha>0$ such that $\left.I\right|_{\partial B_{\rho}(0)} \geq \alpha$;

(iii) For each finite dimensional subspace $E^{\prime} \subset E$, there is $r=r\left(E^{\prime}\right)>0$ such that $I(u) \leq 0$ for $u \in E^{\prime} \backslash B_{r}(0)$, where $B_{r}(0)$ is an open ball in $E$ of radius $r$ centered at 0 .

Then I possesses an unbounded sequence of critical values.

Lemma 3.2. For $u \in E$,

$$
\beta\|u\|_{\infty}^{p} \leq \beta\|u\|_{l^{p}}^{p} \leq\|u\|^{p},
$$

where $\beta=\inf _{t \in \mathbb{Z}} q(t)$.

Lemma 3.3. Assume that (F3) holds. Then for every $(t, x) \in \mathbb{Z} \times \mathbb{R}, s^{-\mu} W(t, s x)$ is nondecreasing on $(0,+\infty)$.

The proof of Lemmas 3.2 and 3.3 is routine and so we omit it.

\section{Proofs of Theorems}

Proof of Theorem 2.1. It is clear that $I(0)=0$. Our proof is devided into three steps.

Step 1 (PS Condition).

Assume that $\left\{u_{k}\right\}_{k \in \mathbb{N}} \subset E$ is a sequence such that $\left\{I\left(u_{k}\right)\right\}_{k \in \mathbb{N}}$ is bounded and $I^{\prime}\left(u_{k}\right) \rightarrow 0$ as $k \rightarrow+\infty$. Then there exists a constant $c>0$ such that

$$
\left|I\left(u_{k}\right)\right| \leq c, \quad\left\|I^{\prime}\left(u_{k}\right)\right\|_{E^{*}} \leq \rho c \quad \text { for } k \in \mathbb{N}
$$


From (2.2), (2.3), (2.4), (4.1), (F3), and (F4), we obtain

$$
\begin{aligned}
p c+p c\left\|u_{k}\right\| \geq & p I\left(u_{k}\right)-\frac{p}{\rho}\left\langle I^{\prime}\left(u_{k}\right), u_{k}\right\rangle \\
= & \frac{\rho-p}{\rho}\left\|u_{k}\right\|^{p}-p \sum_{t \in \mathbb{Z}}\left[W\left(t, u_{k}(t)\right)-\frac{1}{\rho} W_{2}^{\prime}\left(t, u_{k}(t)\right) u_{k}(t)\right] \\
& +p \sum_{t \in \mathbb{Z}} H\left(t, u_{k}(t+n), \ldots, u_{k}(t)\right) \\
& -\frac{p}{\rho} \sum_{t \in \mathbb{Z}} \sum_{i=-n}^{0} H_{2+n+i}^{\prime}\left(t+i, u_{k}(t+n+i), \ldots, u_{k}(t+i)\right) u_{k}(t) \\
= & \frac{\rho-p}{\rho}\left\|u_{k}\right\|^{p}-p \sum_{n \in \mathbb{Z}}\left[W\left(t, u_{k}(t)\right)-\frac{1}{\rho} W_{2}^{\prime}\left(t, u_{k}(t)\right) u_{k}(t)\right] \\
& +p \sum_{t \in \mathbb{Z}} H\left(t, u_{k}(t+n), \ldots, u_{k}(t)\right) \\
& -\frac{p}{\rho} \sum_{t \in \mathbb{Z}} \sum_{i=-n}^{0} H_{2+n+i}^{\prime}\left(t, u_{k}(t+n), \ldots, u_{k}(t)\right) u_{k}(t-i) \\
\geq & \frac{\rho-p}{\rho}\left\|u_{k}\right\|^{p}, k \in \mathbb{N} .
\end{aligned}
$$

By (4.2), there exists a constant $A>0$ such that

$$
\left\|u_{k}\right\| \leq A \text { for } k \in \mathbb{N}
$$

It can be assumed that $u_{k} \rightarrow u_{0}$ in $E$. For any given number $\varepsilon>0$, by (F1), we can choose $\zeta>0$ such that

$$
\begin{aligned}
& |f(t, u(t+n), \ldots, u(t), \ldots, u(t-n))| \\
& \quad \leq \varepsilon \xi^{p-1} \text { for } t \in \mathbb{Z} \backslash J,(u(t+n), \ldots, u(t), \ldots, u(t-n)) \in \mathbb{R}^{n+1},
\end{aligned}
$$

where $\xi=\left(\sum_{i=-n}^{n}|u(t+i)|^{p}\right)^{1 / p} \leq \zeta$.

Since $q(t) \rightarrow \infty$, we can also choose an integer $\Pi>\max \{|k|: k \in J\}$ such that

$$
q(t) \geq \frac{(2 n+1) A^{p}}{\zeta^{p}}, \quad|t| \geq \Pi
$$

By (4.2) and (4.4), we have

$$
\left|u_{k}(t)\right|^{p}=\frac{1}{q(t)} q(t)\left(u_{k}(t)\right)^{p} \leq \frac{\zeta^{p}}{(2 n+1) A^{p}}\left\|u_{k}\right\|^{p} \leq \frac{\zeta^{p}}{2 n+1} \quad \text { for }|t| \geq \Pi, k \in \mathbb{N} .
$$


Since $u_{k} \rightarrow u_{0}$ in $E$, it is easy to verify that $u_{k}(t)$ converges to $u_{0}(t)$ pointwise for all $t \in \mathbb{Z}$, that is

$$
\lim _{k \rightarrow \infty} u_{k}(t)=u_{0}(t), \quad \forall t \in \mathbb{Z}
$$

Hence, we have by (4.6) and (4.7)

$$
\left|u_{0}(t)\right|^{p} \leq \frac{\zeta^{p}}{2 n+1} \text { for }|t| \geq \Pi .
$$

It follows from (4.7) and the continuity of $f(t, u(t+1), \ldots, u(t), \ldots, u(t-n))$ on $u(t+1)$, $\ldots, u(t), \ldots, u(t-n)$ that there exists $k_{0} \in \mathbb{N}$ such that

$$
\sum_{t=-\Pi}^{\Pi}\left|f\left(t, u_{k}(t+n), \ldots, u_{k}(t), \ldots, u_{k}(t-n)\right)-f\left(t, u_{0}(t+n), \ldots, u_{0}(t), \ldots, u_{0}(t-n)\right)\right|<\varepsilon
$$

for $k \geq k_{0}$.

On the other hand, it follows from (F1), (2.4), (4.2), (4.4), (4.6), and (4.8) that

$$
\begin{aligned}
& \sum_{|t|>\Pi}\left|f\left(t, u_{k}(t+n), \ldots, u_{k}(t), \ldots, u_{k}(t-n)\right)-f\left(t, u_{0}(t+n), \ldots, u_{0}(t), \ldots, u_{0}(t-n)\right)\right| \\
& \quad \times\left|u_{k}(t)-u_{0}(t)\right| \\
& \leq \sum_{|t|>\Pi}\left(\left|f\left(t, u_{k}(t+n), \ldots, u_{k}(t), \ldots, u_{k}(t-n)\right)\right|\right. \\
& \left.\quad+\left|f\left(t, u_{0}(t+n), \ldots, u_{0}(t), \ldots, u_{0}(t-n)\right)\right|\right) \times\left(\left|u_{k}(t)\right|+\left|u_{0}(t)\right|\right) \\
& \leq \varepsilon \sum_{|t|>\Pi}\left[\left(\sum_{i=-n}^{n}\left|u_{k}(t+i)\right|^{p-1}\right)+\left(\sum_{i=-n}^{n}\left|u_{0}(t+i)\right|^{p-1}\right)\right]\left(\left|u_{k}(t)\right|+\left|u_{0}(t)\right|\right) \\
& \leq(2 n+1) \varepsilon \sum_{t \in \mathbb{Z}}\left(\left|u_{k}(t)\right|^{p-1}+\left|u_{0}(t)\right|^{p-1}\right)\left(\left|u_{k}(t)\right|+\left|u_{0}(t)\right|\right) \\
& \leq(4 n+2) \varepsilon \sum_{t \in \mathbb{Z}}\left(\left|u_{k}(t)\right|^{p}+\left|u_{0}(t)\right|^{p}\right) \\
& \leq \frac{(4 n+2) \varepsilon}{\beta}\left(A^{p}+\left\|u_{0}\right\|^{p}\right) .
\end{aligned}
$$

Since $\varepsilon$ is arbitrary, combining (4.9) with (4.10), we get

$$
\begin{array}{r}
\sum_{t \in \mathbb{Z}}\left|f\left(t, u_{k}(t+n), \ldots, u_{k}(t), \ldots, u_{k}(t-n)\right)-f\left(t, u_{0}(t+n), \ldots, u_{0}(t), \ldots, u_{0}(t-n)\right)\right| \\
\left|u_{k}(t)-u_{0}(t)\right| \longrightarrow 0 \text { as } k \longrightarrow \infty
\end{array}
$$


Using the Hölder's inequality

$$
a c+b d \leq\left(a^{p}+b^{p}\right)^{1 / p}\left(c^{q}+d^{q}\right)^{1 / q}
$$

where $a, b, c, d$ are nonnegative numbers and $1 / p+1 / q=1, p>1$, it follows from (3.3) and (3.4) that

$$
\begin{aligned}
\left\langle I^{\prime}\left(u_{k}\right)\right. & \left.-I^{\prime}\left(u_{0}\right), u_{k}-u_{0}\right\rangle \\
= & \sum_{t \in \mathbb{Z}}\left|r(t-1) \Delta^{n} u_{k}(t-1)\right|^{p-2}\left(\Delta^{n} u_{k}(t-1), \Delta^{n} u_{k}(t-1)-\Delta^{n} u_{0}(t-1)\right) \\
& +\sum_{t \in \mathbb{Z}} q(t)\left|u_{k}(t)\right|^{p-2}\left(u_{k}(t), u_{k}(t)-u_{0}(t)\right) \\
& -\sum_{t \in \mathbb{Z}}\left|r(t-1) \Delta^{n} u_{0}(t-1)\right|^{p-2}\left(\Delta^{n} u_{0}(t-1), \Delta^{n} u_{k}(t-1)-\Delta^{n} u_{0}(t-1)\right) \\
& -\sum_{t \in \mathbb{Z}} q(t)\left|u_{0}(t)\right|^{p-2}\left(u_{0}(n), u_{k}(t)-u_{0}(t)\right) \\
& -\sum_{t \in \mathbb{Z}}\left(f\left(t, u_{k}(t+n), \ldots, u_{k}(t), \ldots, u_{k}(t-n)\right)\right. \\
= & \left\|u_{k}\right\|^{p}+\left\|u_{0}\right\|^{p}-\sum_{n \in \mathbb{Z}}\left|r(t-1) \Delta^{n} u_{k}(t-1)\right|^{p-2}\left(\Delta^{n} u_{k}(t-1), \Delta^{n} u_{0}(t-1)\right) \\
& -\sum_{t \in \mathbb{Z}} q(t)\left|u_{k}(t)\right|^{p-2}\left(u_{k}(t), u_{0}(t)\right) \\
& -\sum_{t \in \mathbb{Z}}\left|r(t-1) \Delta^{n} u_{0}(t-1)\right|^{p-2}\left(\Delta^{n} u_{0}(t-1), \Delta^{n} u_{k}(t-1)\right) \\
& -\sum_{t \in \mathbb{Z}} q(t)\left|u_{0}(t)\right|^{p-2}\left(u_{0}(t), u_{k}(t)\right) \\
& -\sum_{t \in \mathbb{Z}}\left(f\left(t, u_{k}(t+n), \ldots, u_{k}(t), \ldots, u_{k}(t-n)\right)\right. \\
& -\left(\sum_{t \in \mathbb{Z}}\left|r(t-1) \Delta^{n} u_{k}(t-1)\right|^{p}\right)\left(\sum_{t \in \mathbb{Z}}\left|r(t-1) \Delta^{p} u_{0}(t-1)\right|^{p}\right)^{1 / q} \\
& \left.-f\left(t, u_{0}(t+n), \ldots, u_{0}(t), \ldots, u_{0}(t-n)\right), u_{k}(n)-u_{0}(n)\right) \\
& -\left(\sum_{t \in \mathbb{Z}}\left|r(t-1) \Delta^{n} u_{0}(t-1)\right|^{p}\right)\left(\sum_{t \in \mathbb{Z}}\left|r(t-1) \Delta^{n} u_{k}(t-1)\right|^{p}\right) \\
& \\
& \\
& \\
&
\end{aligned}
$$


Abstract and Applied Analysis

$$
\begin{aligned}
& -\left(\sum_{t \in \mathbb{Z}} q(t)\left|u_{k}(t)\right|^{p}\right)^{1 / p}\left(\sum_{t \in \mathbb{Z}} q(t)\left|u_{0}(t)\right|^{p}\right)^{1 / q} \\
& -\sum_{t \in \mathbb{Z}}\left(f\left(t, u_{k}(t+n), \ldots, u_{k}(t), \ldots, u_{k}(t-n)\right)\right. \\
& \left.-f\left(t, u_{0}(t+n), \ldots, u_{0}(t), \ldots, u_{0}(t-n)\right), u_{k}(t)-u_{0}(t)\right) \\
& \geq\left\|u_{k}\right\|^{p}+\left\|u_{0}\right\|^{p}-\left(\sum_{t \in \mathbb{Z}}\left[\left|r(t-1) \Delta^{n} u_{0}(t-1)\right|^{p}+q(t)\left|u_{0}(t)\right|^{p}\right]\right)^{1 / p} \\
& \cdot\left(\sum_{t \in \mathbb{Z}}\left[\left|r(t-1) \Delta^{n} u_{k}(t-1)\right|^{p}+q(t)\left|u_{k}(t)\right|^{p}\right]\right)^{1 / q} \\
& -\left(\sum_{t \in \mathbb{Z}}\left[\left|r(t-1) \Delta^{n} u_{k}(n-1)\right|^{p}+q(t)\left|u_{k}(t)\right|^{p}\right]\right)^{1 / p} \\
& \cdot\left(\sum_{t \in \mathbb{Z}}\left[\left|r(t-1) \Delta^{n} u_{0}(n-1)\right|^{p}+q(t)\left|u_{0}(t)\right|^{p}\right]\right)^{1 / q} \\
& -\sum_{t \in \mathbb{Z}}\left(f\left(t, u_{k}(t+n), \ldots, u_{k}(t), \ldots, u_{k}(t-n)\right)\right. \\
& \left.-f\left(t, u_{0}(t+n), \ldots, u_{0}(t), \ldots, u_{0}(t-n)\right), u_{k}(t)-u_{0}(t)\right) \\
& =\left\|u_{k}\right\|^{p}+\left\|u_{0}\right\|^{p}-\left\|u_{0}\right\|\left\|u_{k}\right\|^{p-1}-\left\|u_{k}\right\|\left\|u_{0}\right\|^{p-1} \\
& -\sum_{t \in \mathbb{Z}}\left(f\left(t, u_{k}(t+n), \ldots, u_{k}(t), \ldots, u_{k}(t-n)\right)\right. \\
& \left.-f\left(t, u_{0}(t+n), \ldots, u_{0}(t), \ldots, u_{0}(t-n)\right), u_{k}(t)-u_{0}(t)\right) \\
& =\left(\left\|u_{k}\right\|^{p-1}-\left\|u_{0}\right\|^{\mathrm{p}-1}\right)\left(\left\|u_{k}\right\|-\left\|u_{0}\right\|\right) \\
& -\sum_{t \in \mathbb{Z}}\left(f\left(t, u_{k}(t+n), \ldots, u_{k}(t), \ldots, u_{k}(t-n)\right)\right. \\
& \left.-f\left(t, u_{0}(t+n), \ldots, u_{0}(t), \ldots, u_{0}(t-n)\right), u_{k}(t)-u_{0}(t)\right) .
\end{aligned}
$$

Since $I^{\prime}\left(u_{k}\right) \rightarrow 0$ as $k \rightarrow+\infty$ and $u_{k} \rightarrow u_{0}$ in $E$, it follows from (4.11) and (4.13) that

$$
\left\langle I^{\prime}\left(u_{k}\right)-I^{\prime}\left(u_{0}\right), u_{k}-u_{0}\right\rangle \longrightarrow 0 \text { as } k \rightarrow \infty
$$

which yields that $\left\|u_{k}\right\| \rightarrow\|u\|$ as $k \rightarrow+\infty$. By the uniform convexity of $E$ and the fact that $u_{k} \rightarrow u_{0}$ in $E$, it follows from the Kadec-Klee property that $u_{k} \rightarrow u_{0}$ in $E$. Hence, $I$ satisfies (PS)-condition. 
Step 2 (Condition (ii) of Lemma 3.1).

By (F1), there exists $\eta \in(0,1)$ such that

$$
|F(t, u(t+n), \ldots, u(t))| \leq \frac{2^{-p} \beta}{4 p(n+1)} \sum_{i=0}^{n}|u(t+i)|^{p} \quad \text { for } t \in \mathbb{Z} \backslash J,\left(\sum_{i=0}^{n}|u(t+i)|^{p}\right)^{1 / p} \leq \eta \text {. }
$$

Set

$$
M=\sup \{W(t, u)|t \in J, u \in \mathbb{R},| u \mid=1\},
$$

and $\delta=\min \left\{(\beta /(4 p M+1))^{(\mu-p)}, \eta\right\}$. If $\|u\|=\beta^{1 / p} \delta:=\rho$, then by Lemma 3.2, $|u(t)| \leq \delta \leq \eta<1$ for $t \in \mathbb{Z}$. By (4.16) and Lemma 3.2, we have

$$
\begin{aligned}
\sum_{t \in J} W(t, u(t)) & \leq \sum_{t \in J, u(t) \neq 0} W\left(t, \frac{u(t)}{|u(t)|}\right)|u(t)|^{\mu} \\
& \leq M \sum_{t \in J}|u(t)|^{\mu} \\
& \leq M \delta^{\mu-p} \sum_{t \in J}|u(t)|^{p} \\
& \leq \frac{M \delta^{\mu-p}}{\beta} \sum_{t \in J} q(t)|u(t)|^{p} \\
& \leq \frac{1}{4 p} \sum_{t \in J} q(t)|u(t)|^{p}
\end{aligned}
$$

Set $\alpha=\beta \delta^{p} / 2 p$. Hence, from (3.3), (4.15), (4.17), (q), (F1), and (F2), we have

$$
\begin{aligned}
I(u)= & \frac{1}{p}\|u\|^{p}-\sum_{t \in \mathbb{Z}} F(t, u(t+n), \ldots, u(t)) \\
= & \frac{1}{p}\|u\|^{p}-\sum_{t \in \mathbb{Z} \backslash J} F(t, u(t+n), \ldots, u(t))-\sum_{t \in J} F(t, u(t+n), \ldots, u(t)) \\
\geq & \frac{1}{p}\|u\|^{p}-\frac{2^{-p} \beta}{4 p(n+1)} \sum_{t \in \mathbb{Z} \backslash J}\left(\sum_{i=0}^{n}|u(t+i)|^{p}\right)-\sum_{t \in J} W(t, u(t)) \\
& +\sum_{t \in J} H(t, u(\mathrm{t}+n), \ldots, u(t)) \\
\geq & \frac{1}{p}\|u\|^{p}-\frac{\beta}{4 p} \sum_{t \in \mathbb{Z}}|u(t)|^{p}-\frac{1}{4 p} \sum_{t \in J} q(t)|u(t)|^{p} \\
\geq & \frac{1}{p}\|u\|^{p}-\frac{1}{4 p} \sum_{t \in \mathbb{Z}} q(t)|u(t)|^{p}-\frac{1}{4 p} \sum_{t \in J} q(t)|u(t)|^{p}
\end{aligned}
$$


Abstract and Applied Analysis

$$
\begin{aligned}
& \geq \frac{1}{p}\|u\|^{p}-\frac{1}{4 p}\|u\|^{p}-\frac{1}{4 p}\|u\|^{p} \\
& =\frac{1}{2 p}\|u\|^{p} \\
& =\alpha .
\end{aligned}
$$

equation (4.18) shows that $\|u\|=\rho$ implies that $I(u) \geq \alpha$, that is, $I$ satisfies assumption (ii) of Lemma 3.1.

Step 3 (Condition (iii) of Lemma 3.1).

Let $E^{\prime}$ be a finite dimensional subspace of $E$. Assume that $\operatorname{dim} E^{\prime}=m$ and $u_{1}, u_{2}, \ldots, u_{m}$ is a base of $E^{\prime}$.

For any $u \in E^{\prime}$, there exist $\lambda_{i} \in \mathbb{R}, i=1,2, \ldots, m$ such that

$$
u(t)=\sum_{i=1}^{m} \lambda_{i} u_{i}(t) \quad \text { for } t \in \mathbb{Z}
$$

Let

$$
\|u\|_{*}=\sum_{i=1}^{m}\left|\lambda_{i}\right|\left\|u_{i}\right\|
$$

It is easy to verify that $\|\cdot\|_{*}$ defined by (4.20) is a norm of $E^{\prime}$. Since all the norms of a finite dimensional normed space are equivalent, so there exists a constant $d>0$ such that

$$
\|u\|_{*} \leq d\|u\|_{\infty} \text { for } u \in E^{\prime}
$$

Assume that

$$
\left\|u_{i}\right\|=d, \quad i=1,2, \ldots, m \text {. }
$$

Since $u_{i} \in E$, by Lemma 3.2, we can choose an integer $\Pi_{1}>0$ such that

$$
\left|u_{i}(t)\right|<\frac{\eta}{m}, \quad i=1,2, \ldots, m,|t|>\Pi_{1}
$$

where $\eta$ is given in (4.15). Set

$$
\Theta=\left\{\sum_{i=1}^{m} \lambda_{i} u_{i}(t): \lambda_{i} \in \mathbb{R}, i=1,2, \ldots, m ; \sum_{i=1}^{m}\left|\lambda_{i}\right|=1\right\}=\left\{u \in E^{\prime}:\|u\|_{*}=d\right\} .
$$


Hence, for $u \in \Theta$, let $t_{0}=t_{0}(u) \in \mathbb{Z}$ such that

$$
\left|u\left(t_{0}\right)\right|=\|u\|_{\infty}
$$

Then, by (4.19)-(4.25), we have

$$
\begin{aligned}
d & =d \sum_{i=1}^{m}\left|\lambda_{i}\right|=\sum_{i=1}^{m}\left|\lambda_{i}\right|\left\|u_{i}\right\|=\|u\|_{*} \\
& \leq d\|u\|_{\infty}=d\left|u\left(t_{0}\right)\right| \\
& \leq d \sum_{i=1}^{m}\left|\lambda_{i}\right| \| u_{i}\left(t_{0}\right) \mid, \quad u \in \Theta .
\end{aligned}
$$

This shows that $\left|u\left(t_{0}\right)\right| \geq 1$ and there exists $i_{0} \in\{1,2, \ldots, m\}$ such that $\left|u_{i_{0}}\left(t_{0}\right)\right| \geq 1 / m$, which, together with (4.23), implies that $\left|t_{0}\right| \leq \Pi_{1}$.

Set

$$
\tau=\min \left\{W(t, x):|t| \leq \Pi_{1},|x|=1\right\} .
$$

Since $W(t, x)>0$ for all $t \in \mathbb{Z}$ and $x \in \mathbb{R} \backslash\{0\}$, and $W(t, x)$ is continuous in $x$, so $\tau>0$. It follows from (4.27) and Lemma 3.3 that

$$
\begin{aligned}
\sum_{n=-\Pi_{1}}^{\Pi_{1}} W(t, u(t)) & \geq W\left(t_{0}, u\left(t_{0}\right)\right) \\
& \geq W\left(t_{0}, \frac{u\left(t_{0}\right)}{\left|u\left(t_{0}\right)\right|}\right)\left|u\left(t_{0}\right)\right|^{\mu} \\
& \geq\left[\min _{|x|=1} W\left(t_{0}, x\right)\right]\left|u\left(t_{0}\right)\right|^{\mu} \\
& \geq \tau \quad \text { for } u \in \Theta .
\end{aligned}
$$

For any $u \in E$, it follows from (F5) that

$$
\begin{aligned}
& \sum_{t=-\Pi_{1}}^{\Pi_{1}} H(t, u(t+n), \ldots, u(t)) \\
& =\sum_{\left\{t \in \mathbb{Z}\left(-\Pi_{1}, \Pi_{1}\right),\left(\sum_{i=0}^{n}|u(t+i)|^{p}\right)^{1 / p}>1\right\}} H(t, u(t+n), \ldots, u(t)) \\
& \quad+\sum_{\left\{t \in \mathbb{Z}\left(-\Pi_{1}, \Pi_{1}\right),\left(\sum_{i=0}^{n}|u(t+i)|^{p}\right)^{1 / p}\right\} \leq 1} H(t, u(t+n), \ldots, u(t))
\end{aligned}
$$


Abstract and Applied Analysis

$$
\begin{aligned}
& \leq b \sum_{\left\{t \in \mathbb{Z}\left(-\Pi_{1}, \Pi_{1}\right),\left(\sum_{i=0}^{n}|u(t+i)|^{p}\right)^{1 / p}>1\right\}}\left(\sum_{i=0}^{n}|u(t+i)|^{p}\right)^{\rho / p} \\
& +\sum_{t=-\Pi_{1}\left(\sum_{i=0}^{n}|u(t+i)|^{p}\right)^{1 / p} \leq 1}^{\Pi_{1}} H(t, u(t+n), \ldots, u(t))
\end{aligned}
$$

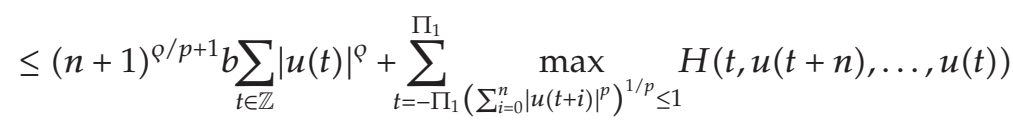

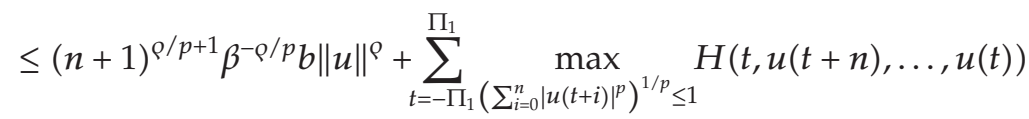

$$
\begin{aligned}
& =M_{1}\|u\|^{\rho}+M_{2}
\end{aligned}
$$

where

$$
M_{1}=(n+1)^{\varrho / p+1} \beta^{-\varrho / p} b, \quad M_{2}=\sum_{t=-\Pi_{1}\left(\sum_{i=0}^{n}|u(t+i)|^{p}\right)^{1 / p} \leq 1}^{\Pi_{1}} H(t, u(t+n), \ldots, u(t)) .
$$

From (3.3), (3.7), (4.28), and (4.29), we have for $u \in \Theta$ and $\sigma>1$

$$
\begin{aligned}
I(\sigma u) & =\frac{\sigma^{p}}{p}\|u\|^{p}-\sum_{t \in \mathbb{Z}} F(t, \sigma u(t+n), \ldots, \sigma u(t)) \\
& =\frac{\sigma^{p}}{p}\|u\|^{p}-\sum_{|t|>\Pi_{2}} F(t, \sigma u(t+n), \ldots, \sigma u(t))-\sum_{|t| \leq \Pi_{2}} F(t, \sigma u(t+n), \ldots, \sigma u(t)) \\
& \leq \frac{\sigma^{p}}{p}\|u\|^{p}+\sum_{|t|>\Pi_{2}} \sum_{i=0}^{n} \frac{\beta \sigma^{p}}{4 p(n+1)}|u(t+i)|^{p}-\sum_{|t| \leq \Pi_{2}} F(t, \sigma u(t+n), \ldots, \sigma u(t)) \\
& \leq \frac{\sigma^{p}}{p}\|u\|^{p}+\frac{\sigma^{p}}{4 p}\|u\|^{p}-\sum_{|t| \leq \Pi_{2}} F(t, \sigma u(t+n), \ldots, \sigma u(t)) \\
& =\frac{\sigma^{p}}{p}\|u\|^{p}+\frac{\sigma^{p}}{4 p}\|u\|^{p}-\sum_{|t| \leq \Pi_{2}} W(t, \sigma u(t))+\sum_{|t| \leq \Pi_{2}} H(t, \sigma u(t+n), \ldots, \sigma u(t)) \\
& \leq \frac{\sigma^{p}}{p}\|u\|^{p}+\frac{\sigma^{p}}{4 p}\|u\|^{p}+\sigma^{\rho}\left(M_{1}\|u\|^{\rho}+M_{2}\right)-\tau \sigma^{\mu} \\
& =\frac{(c \sigma)^{p}}{p}+\frac{c^{p} \sigma^{p}}{4 p}+M_{1}(c \sigma)^{\rho}+M_{2} \sigma^{\rho}-\tau \sigma^{\mu} .
\end{aligned}
$$


Since $\mu>\rho>p$, we deduce that there is $\sigma_{0}=\sigma_{0}\left(d, M_{1}, M_{2}, \tau\right)=\sigma_{0}\left(E^{\prime}\right)>1$ such that

$$
I(\sigma u)<0 \text { for } u \in \Theta, \sigma \geq \sigma_{0} .
$$

That is

$$
I(u)<0 \quad \text { for } u \in E^{\prime},\|u\| \geq d \sigma_{0} .
$$

This shows that (iii) of Lemma 3.1 holds. By Lemma 3.1, I possesses an unbounded sequence $\left\{d_{k}\right\}_{k \in \mathbb{N}}$ of critical values with $d_{k}=I\left(u_{k}\right)$, where $u_{k}$ is such that $I^{\prime}\left(u_{k}\right)=0$ for $k=1,2, \ldots$ If $\left\{\left\|u_{k}\right\|\right\}_{k \in \mathbb{N}}$ is bounded, then there exists $B>0$ such that

$$
\left\|u_{k}\right\| \leq B \quad \text { for } k \in \mathbb{N} \text {. }
$$

By a similar fashion for the proof of (4.15), for the given $\eta$ in (4.15), there exists $\Pi_{3}>0$ such that

$$
\left|u_{k}(t)\right| \leq \eta \quad \text { for }|t| \geq \Pi_{3}, k \in \mathbb{N}
$$

Thus, from (F2), (3.7), (4.34), and (4.35), we have

$$
\begin{aligned}
\frac{1}{p}\left\|u_{k}\right\|^{p} & =d_{k}+\sum_{t \in \mathbb{Z}} F\left(t, u_{k}(t+n), \ldots, u_{k}(t)\right) \\
& =d_{k}+\sum_{|t|>\Pi_{3}} F\left(t, u_{k}(t+n), \ldots, u_{k}(t)\right)+\sum_{t=-\Pi_{3}}^{\Pi_{3}} F\left(t, u_{k}(t+n), \ldots, u_{k}(t)\right) \\
& \geq d_{k}-\frac{\beta}{4 p(n+1)} \sum_{|t|>\Pi_{3}} \sum_{i=0}^{n}\left|u_{k}(t+i)\right|^{p}-\sum_{n=-\Pi_{3}}^{\Pi_{3}} H\left(t, u_{k}(t+n), \ldots, u_{k}(t)\right) \\
& \geq d_{k}-\frac{1}{4 p}\left\|u_{k}\right\|^{p}-\sum_{n=-\Pi_{3}\left|u_{k}\right| \leq B / \sqrt{\beta}}^{\Pi_{3}} \max _{\mid}\left|H\left(t, u_{k}(t+n), \ldots, u_{k}(t)\right)\right| .
\end{aligned}
$$

It follows that

$$
d_{k} \leq \frac{5}{4 p}\left\|u_{k}\right\|^{p}+\sum_{t=-\Pi_{3}}^{\Pi_{3}} \max _{\left|u_{k}\right| \leq B / \sqrt{\beta}}\left|H\left(t, u_{k}(t+n), \ldots, u_{k}(t)\right)\right|<+\infty
$$

This contradicts the fact that $\left\{d_{k}\right\}_{k \in \mathbb{N}}$ is unbounded, and so $\left\{\left\|u_{k}\right\|\right\}_{k \in \mathbb{N}}$ is unbounded.

Proof of Theorem 2.2. In the proof of Theorem 2.1, the condition that $H(t, u(t+n), \ldots, u(t)) \geq 0$ for $(t, u(t+n), \ldots, u(t)) \in J \times \mathbb{R}^{n+1}, \gamma=\left(\sum_{i=0}^{n}|\mathrm{u}(t+i)|^{p}\right)^{1 / p} \leq 1$ in (F1) is only used in the proofs 
of assumption (ii) of Lemma 3.1. Therefore, we only prove that assumption (ii) of Lemma 3.1 still holds using (F2') instead of (F2). By (F2'), it follows that

$$
|F(t, u(t+n), \ldots, u(t))| \leq \frac{2^{-p} \beta}{2 p(n+1)}\left(\sum_{i=0}^{n}|u(t+i)|^{p}\right) \quad \text { for } t \in \mathbb{Z},\left(\sum_{i=0}^{n}|u(t+i)|^{p}\right)^{1 / p} \leq \eta
$$

If $\|u\|=\beta^{1 / p} \eta:=\rho$, then by Lemma $3.2,|u(t)| \leq \eta$ for $t \in \mathbb{Z}$. Set $\alpha=\beta \eta^{p} / 2 p$. Hence, from (3.3), (4.38), and Lemma 3.2, we have

$$
\begin{aligned}
I(u) & =\frac{1}{p}\|u\|^{p}-\sum_{t \in \mathbb{Z}} F(t, u(t+n), \ldots, u(t)) \\
& \geq \frac{1}{p}\|u\|^{p}-\frac{2^{-p} \beta}{2 p(n+1)}\left(\sum_{i=0}^{n}|u(t+i)|^{p}\right) \\
& \geq \frac{1}{p}\|u\|^{p}-\frac{1}{2 p}\|u\|^{p} \\
& =\frac{1}{2 p}\|u\|^{p} \\
& =\alpha .
\end{aligned}
$$

equation (4.39) shows that $\|u\|=\rho$ implies that $I(u) \geq \alpha$, that is, assumption (ii) of Lemma 3.1 holds. The proof of Theorem 2.2 is completed.

Proof of Theorem 2.3. We first show that $I$ satisfies condition (C). Assume that $\left\{u_{k}\right\}_{k \in \mathbb{N}} \subset E$ is a (C) sequence of $I$, that is, $\left\{I\left(u_{k}\right)\right\}_{k \in \mathbb{N}}$ is bounded and $\left(1+\left\|u_{k}\right\|\right)\left\|I^{\prime}\left(u_{k}\right)\right\| \rightarrow 0$ as $k \rightarrow+\infty$. Then it follows from (3.3) and (3.4) that

$$
\begin{aligned}
C_{1} & \geq p I\left(u_{k}\right)-\left\langle I^{\prime}\left(u_{k}\right), u_{k}\right\rangle \\
& =\sum_{t \in \mathbb{Z}}\left[\sum_{i=-n}^{0} F_{2+n+i}^{\prime}\left(t, u_{k}(t+n), \ldots, u_{k}(t)\right) u_{k}(t-i)-p F\left(t, u_{k}(t+n), \ldots, u_{k}(t)\right)\right] .
\end{aligned}
$$

It follows from (F8) that there exists $\eta \in(0,1)$ such that (4.15) holds. By (F7) and (F8), we have

$$
\sum_{i=-n}^{0} F_{2+n+i}^{\prime}(t, u(t+n), \ldots, u(t)) u(t-i)>p F\left(t, u_{k}(t+n), \ldots, u(t)\right) \geq 0
$$


and for $t \in \mathbb{Z}, \sum_{i=0}^{n}|u(t+i)|^{p} \geq \eta^{p}$, we have

$$
\begin{aligned}
& F\left(t, u_{k}(t+n), \ldots, u_{k}(t)\right) \leq\left[a+b\left(\sum_{i=0}^{n}|u(t+i)|^{p}\right)^{v / p}\right] \\
& \quad \times\left[\sum_{i=-n}^{0} F_{2+n+i}^{\prime}\left(t, u_{k}(t+n), \ldots, u_{k}(t)\right) u_{k}(t-i)-p F\left(t, u_{k}(t+n), \ldots, u_{k}(t)\right)\right] .
\end{aligned}
$$

It follows from (3.2), (3.3), (4.38), (4.40), (4.41), and (4.42) that

$$
\begin{aligned}
& \frac{1}{p}\left\|u_{k}\right\|^{p}=I\left(u_{k}\right)+\sum_{t \in \mathbb{Z}} F\left(t, u_{k}(t+n), \ldots, u(t)\right) \\
& =I\left(u_{k}\right)+\sum_{t \in \mathbb{Z}\left(\left(\sum_{i=0}^{n}|u(t+i)|^{p}\right)^{1 / p} \leq \eta\right)} F\left(t, u_{k}(t+n), \ldots, u(t)\right) \\
& +\sum_{t \in \mathbb{Z}\left(\left(\sum_{i=0}^{n}|u(t+i)|^{p}\right)^{1 / p}>\eta\right)} F\left(t, u_{k}(t+n), \ldots, u(t)\right) \\
& \leq I\left(u_{k}\right)+\frac{2^{-p} \beta}{2 p(n+1)} \sum_{t \in \mathbb{Z}\left(\left(\sum_{i=0}^{n}|u(t+i)|^{p}\right)^{1 / p} \leq \eta\right)}\left|u_{k}(t+i)\right|^{p} \\
& +\sum_{t \in \mathbb{Z}\left(\left(\sum_{i=0}^{n}|u(t+i)|^{p}\right)^{1 / p}>\eta\right)}\left[a+b\left(\sum_{i=0}^{n}|u(t+i)|^{p}\right)^{v / p}\right] \\
& \times\left[\sum_{i=-n}^{0} F_{2+n+i}^{\prime}\left(t, u_{k}(t+n), \ldots, u_{k}(t)\right) u_{k}(t-i)-p F\left(t, u_{k}(t+n), \ldots, u_{k}(t)\right)\right] \\
& \leq C_{2}+\frac{1}{2 p}\left\|u_{k}\right\|^{2}+\sum_{t \in \mathbb{Z}}\left[a+b\left(\sum_{i=0}^{n}|u(t+i)|^{p}\right)^{v / p}\right] \\
& \times\left[\sum_{i=-n}^{0} F_{2+n+i}^{\prime}\left(t, u_{k}(t+n), \ldots, u_{k}(t)\right) u_{k}(t-i)-p F\left(t, u_{k}(t+n), \ldots, u_{k}(t)\right)\right] \\
& \leq C_{2}+\frac{1}{2 p}\left\|u_{k}\right\|^{p}+\left(a+b\left\|u_{k}\right\|_{\infty}^{v}\right) \sum_{t \in \mathbb{Z}}\left[\left(\nabla W\left(n, u_{k}(n)\right), u_{k}(n)\right)-p W\left(n, u_{k}(n)\right)\right] \\
& \leq C_{2}+\frac{1}{2 p}\left\|u_{k}\right\|^{p}+C_{1}\left(a+b(n+1)\left\|u_{k}\right\|_{\infty}^{v}\right) \\
& \leq C_{2}+\frac{1}{2 p}\left\|u_{k}\right\|^{p}+C_{1}\left\{a+\beta^{-v / p} b(n+1)\left\|u_{k}\right\|^{v}\right\}, \quad k \in \mathbb{N} .
\end{aligned}
$$

Since $v<p$, it follows from (4.43) that $\left\{\left\|u_{k}\right\|\right\}_{k \in \mathbb{N}}$ is bounded. Similar to the proof of Theorem 2.1, we can prove that $\left\{u_{k}\right\}$ has a convergent subsequence in $E$. Hence, $I$ satisfies condition (C).

It is obvious that $I$ is even and $I(0)=0$, and so assumption (i) of Lemma 3.1 holds. The proof of assumption (ii) of Lemma 3.1 is the same as in the proof of Theorem 2.2. 
Finally, it remains to show that $I$ satisfies assumption (iii) of Lemma 3.1. Let $E^{\prime}$ be a finite dimensional subspace of $E$. Since all norms of a finite dimensional normed space are equivalent, so there is a constant $d>0$ such that (4.21) holds. Assume that $\operatorname{dim} E^{\prime}=m$ and $u_{1}, u_{2}, \ldots, u_{m}$ are the bases of $E^{\prime}$ such that (4.22) holds. Let $\eta, \Pi_{1}$, and $\Theta$ be the same as in the proof of Theorem 2.1. Then (4.23), (4.25), and (4.26) hold. For the $\Pi_{1}$ given in the proof of Theorem 2.1. By (F9), there exists $\sigma_{0}=\sigma_{0}\left(d, \Pi_{1}\right)>1$ such that

$$
s^{-p} \min _{|x|=1} W(n, s x) \geq(2 d)^{p} \quad \text { for } s \geq \frac{\sigma_{0}}{2}, n \in \mathbb{Z}\left(-\Pi_{1}, \Pi_{1}\right)
$$

It follows from (F7), (F9), (3.3), and (4.44) that

$$
\begin{aligned}
I(\sigma u) & =\frac{\sigma^{p}}{p}\|u\|^{p}-\sum_{t \in \mathbb{Z}} F(t, \sigma u(t+n), \ldots, \sigma u(t)) \\
& \leq \frac{\sigma^{p}}{p}\|u\|^{p}-F\left(t_{0}, \sigma u\left(t_{0}\right)\right) \\
& \leq \frac{\sigma^{p}}{p}\|u\|^{p}-\min _{|x|=1} F\left(t_{0}, \sigma\left|u\left(t_{0}\right)\right| x\right) \\
& \leq \frac{(d \sigma)^{p}}{p}-(d \sigma)^{p}\left|u\left(t_{0}\right)\right|^{p} \\
& \leq \frac{(d \sigma)^{p}}{p}-(d \sigma)^{p} \\
& =-\frac{(d \sigma)^{p}}{q}, \quad u \in \Theta, \sigma \geq \sigma_{0} .
\end{aligned}
$$

We deduce that there is $\sigma_{0}=\sigma_{0}\left(d, \Pi_{1}\right)=\sigma_{0}\left(E^{\prime}\right)>1$ such that

$$
I(\sigma u)<0 \text { for } u \in \Theta, \sigma \geq \sigma_{0} .
$$

That is

$$
I(u)<0 \quad \text { for } u \in E^{\prime},\|u\| \geq d \sigma_{0} .
$$

This shows that condition (iii) of Lemma 3.1 holds. By Lemma 3.1, I possesses an unbounded sequence $\left\{d_{k}\right\}_{k \in \mathbb{N}}$ of critical values with $d_{k}=I\left(u_{k}\right)$, where $u_{k}$ is such that $I^{\prime}\left(u_{k}\right)=0$ for $k=1,2, \ldots$. Since

$$
\frac{1}{p}\left\|u_{k}\right\|^{p}=d_{k}+\sum_{n \in \mathbb{Z}} F\left(t, u_{k}(t+n), \ldots, u_{k}(t)\right) \geq d_{k}
$$

and $\left\{d_{k}\right\}_{k \in \mathbb{N}}$ is unbounded, it follows that $\left\{\left\|u_{k}\right\|\right\}_{k \in \mathbb{N}}$ is unbounded. The proof is complete. 


\section{Examples}

In this section, we give some examples to illustrate our results.

Example 5.1. In (1.1), let $q(t) \rightarrow+\infty$ as $|t| \rightarrow+\infty$ and

$$
F(t, u(t+n), \ldots, u(t))=\left[|u(t)|^{4+|t|}-\left(\sum_{i=0}^{n} u^{2}(t+i)\right)^{(5+3|t|) / 2(4+|t|)}\right] .
$$

Let $p=2, \mu=4, \varrho=3, J=\{-3,-2,-1,0,1,2,3\}$ and

$$
W(t, u(t))=|u(t)|^{4+|t|}, \quad H(t, u(t+n), \ldots, u(t))=\left(\sum_{i=0}^{n} u^{2}(t+i)\right)^{(5+3|t|) / 2(4+|t|)} .
$$

Then it is easy to verify that all conditions of Theorem 2.1 are satisfied. By Theorem 2.1, (1.1) has a nontrivial homoclinic solution.

Example 5.2. In (1.1), let $r(t)>0, q(t) \rightarrow+\infty$ as $|t| \rightarrow+\infty$ and

$$
F(t, u(t+n), \ldots, u(t))=\left(\sum_{k=1}^{m_{1}} a_{k}|u(t)|^{\mu_{k}}-\sum_{j=1}^{m_{2}} b_{j}\left(\sum_{i=0}^{n}|u(t+i)|^{p}\right)^{Q_{j} / p}\right),
$$

where $\mu_{1}>\mu_{2}>\cdots>\mu_{m_{1}}>\rho_{1}>\rho_{2}>\cdots>\rho_{m_{2}}>p, a_{k}, b_{j}>0, k=1,2, \ldots, m_{1} ; j=1,2, \ldots, m_{2}$. Let $\mu=\mu_{m_{1}}, Q=\rho_{1}$, and

$$
W(t, u(t))=\sum_{k=1}^{m_{1}} a_{k}|u(t)|^{\mu_{k}}, \quad H(t, u(t+n), \ldots, u(t))=\sum_{j=1}^{m_{2}} b_{j}\left(\sum_{i=0}^{n}|u(t+i)|^{p}\right)^{\rho_{j} / p}
$$

Then it is easy to verify that all conditions of Theorem 2.2 are satisfied. By Theorem 2.2, (1.1) has a nontrivial homoclinic solution.

Example 5.3. In (1.1), let

$$
F(t, u(t+n), \ldots, u(t))=q(t) \sum_{i=0}^{n}|u(t+i)|^{p} \ln \left[1+\left(\sum_{i=0}^{n}|u(t+i)|^{p}\right)^{1 / p}\right],
$$

where $q: \mathbb{Z} \rightarrow(0, \infty)$ such that $q(t) \rightarrow+\infty$ as $|t| \rightarrow+\infty$. Since

$$
\begin{aligned}
& \sum_{i=-n}^{0} F_{2+n+i}^{\prime}(t, u(t+n), \ldots, u(t)) u(t-i) \\
& \quad=q(t)\left[p \sum_{i=0}^{n}|u(t+i)|^{p} \ln \left[1+\left(\sum_{i=0}^{n}|u(t+i)|^{p}\right)^{1 / p}\right]+\frac{\left(\sum_{i=0}^{n}|u(t+i)|^{p}\right)^{p+1 / p}}{1+\left(\sum_{i=0}^{n}|u(t+i)|^{p}\right)^{1 / p}}\right]
\end{aligned}
$$




$$
\begin{aligned}
& \geq\left(p+\frac{1}{1+\left(\sum_{i=0}^{n}|u(t+i)|^{p}\right)^{1 / p}}\right) F(t, u(t+n), \ldots, u(t)) \\
& \geq 0, \quad \forall(t, u(t+n), \ldots, u(t)) \in \mathbb{Z} \times \mathbb{R}^{n+1} .
\end{aligned}
$$

This shows that (F8) holds with $a=b=v=1$. It is easy to verify that assumptions (q), (F1), and (F7) of Theorem 2.3 are satisfied. By Theorem 2.3, (1.1) has an unbounded sequence of homoclinic solutions.

\section{Acknowledgment}

This work is partially supported by Major Project of Science Research Fund of Education Department in Hunan (no: 11A095).

\section{References}

[1] P. Chen and H. Fang, "Existence of periodic and subharmonic solutions for second-order $p$-Laplacian difference equations," Advances in Difference Equations, vol. 2007, Article ID 42530, 9 pages, 2007.

[2] P. Chen and X. Tang, "Existence of homoclinic orbits for $2 n$ th-order nonlinear difference equations containing both many advances and retardations," Journal of Mathematical Analysis and Applications, vol. 381, no. 2, pp. 485-505, 2011.

[3] P. Chen and X. H. Tang, "Existence of infinitely many homoclinic orbits for fourth-order difference systems containing both advance and retardation," Applied Mathematics and Computation, vol. 217, no. 9, pp. 4408-4415, 2011.

[4] P. Chen and L. Xiao, "Existence of homoclinic orbit for second-order nonlinear difference equation," Electronic Journal of Qualitative Theory of Differential Equations, vol. 72, pp. 1-14, 2010.

[5] P. Chen and X. H. Tang, "Existence of homoclinic solutions for the second-order discrete p-Laplacian systems," Taiwanese Journal of Mathematics, vol. 15, no. 5, pp. 2123-2143, 2011.

[6] P. Chen and X. H. Tang, "New existence of homoclinic orbits for a second-order Hamiltonian system," Computers \& Mathematics with Applications, vol. 62, no. 1, pp. 131-141, 2011.

[7] M. Izydorek and J. Janczewska, "Homoclinic solutions for a class of the second order Hamiltonian systems," Journal of Differential Equations, vol. 219, no. 2, pp. 375-389, 2005.

[8] M. Ma and Z. Guo, "Homoclinic orbits and subharmonics for nonlinear second order difference equations," Nonlinear Analysis. Theory, Methods \& Applications, vol. 67, no. 6, pp. 1737-1745, 2007.

[9] M. Ma and Z. Guo, "Homoclinic orbits for second order self-adjoint difference equations," Journal of Mathematical Analysis and Applications, vol. 323, no. 1, pp. 513-521, 2006.

[10] W. Omana and M. Willem, "Homoclinic orbits for a class of Hamiltonian systems," Differential and Integral Equations, vol. 5, no. 5, pp. 1115-1120, 1992.

[11] Z. Q. Ou and C. L. Tang, "Existence of homoclinic orbits for the second order Hamiltonian systems," Journal of Mathematical Analysis and Applications, vol. 291, no. 1, pp. 203-213, 2004.

[12] P. H. Rabinowitz, "Homoclinic orbits for a class of Hamiltonian systems," Proceedings of the Royal Society of Edinburgh, vol. 114, no. 1-2, pp. 33-38, 1990.

[13] P. H. Rabinowitz and K. Tanaka, "Some results on connecting orbits for a class of Hamiltonian systems," Mathematische Zeitschrift, vol. 206, no. 3, pp. 473-499, 1991.

[14] X. H. Tang and X. Lin, "Existence of infinitely many homoclinic orbits in discrete Hamiltonian systems," Journal of Mathematical Analysis and Applications, vol. 354, no. 2, pp. 539-549, 2009.

[15] J. Yu, H. Shi, and Z. Guo, "Homoclinic orbits for nonlinear difference equations containing both advance and retardation," Journal of Mathematical Analysis and Applications, vol. 352, no. 2, pp. 799-806, 2009.

[16] Z. Guo and J. Yu, "Periodic and subharmonic solutions for superquadratic discrete Hamiltonian systems," Nonlinear Analysis. Theory, Methods E Applications, vol. 55, no. 7-8, pp. 969-983, 2003. 
[17] Z. Guo and J. Yu, "The existence of periodic and subharmonic solutions of subquadratic second order difference equations," Journal of the London Mathematical Society, vol. 68, no. 2, pp. 419-430, 2003.

[18] J. Rodriguez and D. L. Etheridge, "Periodic solutions of nonlinear second-order difference equations," Advances in Difference Equations, vol. 2005, no. 2, pp. 173-192, 2005.

[19] R. P. Agarwal, Difference Equations and Inequalities, vol. 228, Marcel Dekker, New York, NY, USA, 2nd edition, 2000.

[20] C. D. Ahlbrandt and A. Peterson, "The $(n, n)$-disconjugacy of a $2 n$th order linear difference equation," Computers \& Mathematics with Applications, vol. 28, no. 1-3, pp. 1-9, 1994.

[21] Z. Zhou, J. Yu, and Z. Guo, "Periodic solutions of higher-dimensional discrete systems," Proceedings of the Royal Society of Edinburgh, vol. 134, no. 5, pp. 1013-1022, 2004.

[22] D. Smets and M. Willem, "Solitary waves with prescribed speed on infinite lattices," Journal of Functional Analysis, vol. 149, no. 1, pp. 266-275, 1997. 


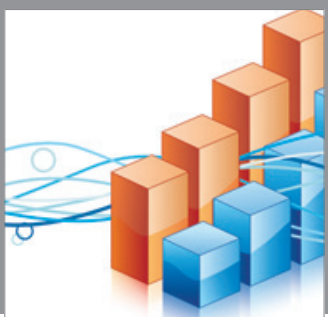

Advances in

Operations Research

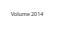

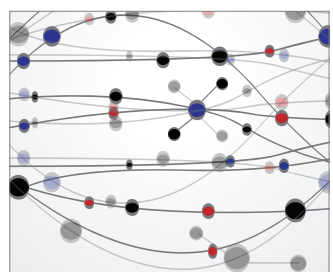

\section{The Scientific} World Journal
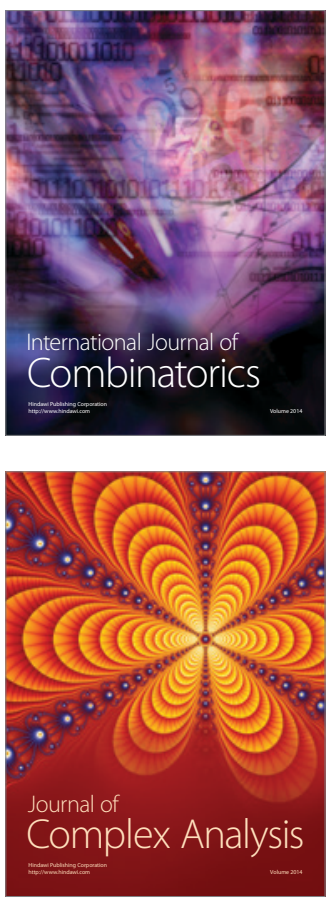

International Journal of

Mathematics and

Mathematical

Sciences
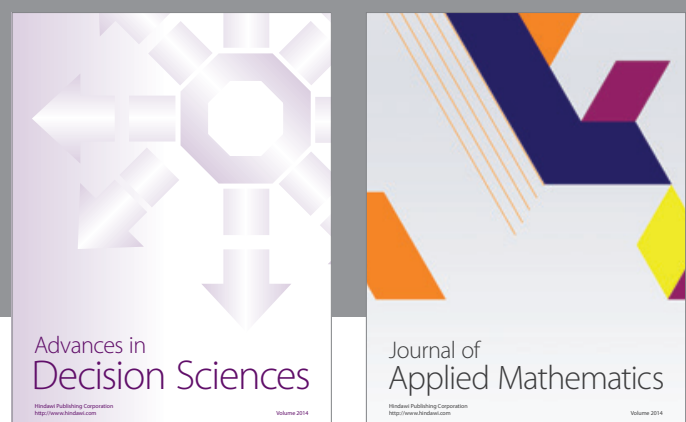

Journal of

Applied Mathematics
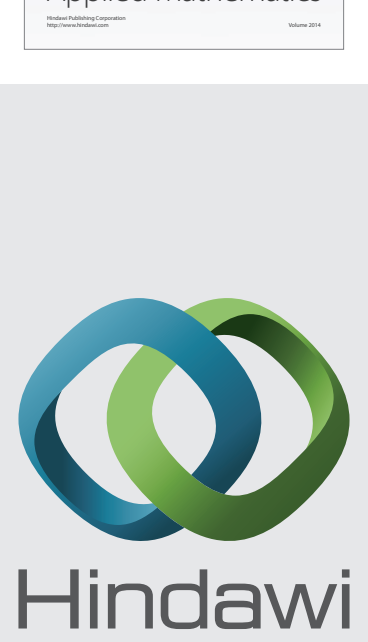

Submit your manuscripts at http://www.hindawi.com
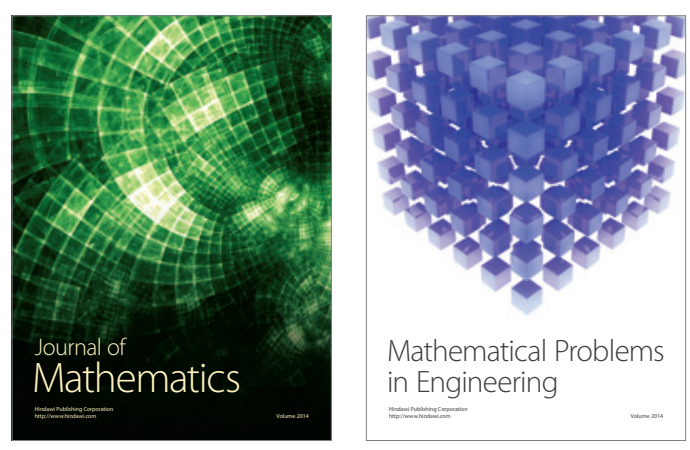

Mathematical Problems in Engineering
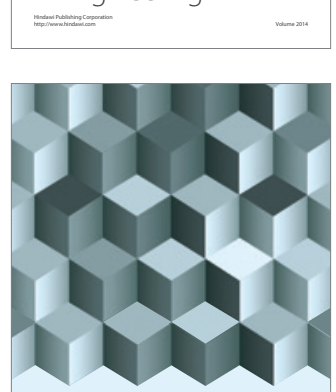

Journal of

Function Spaces
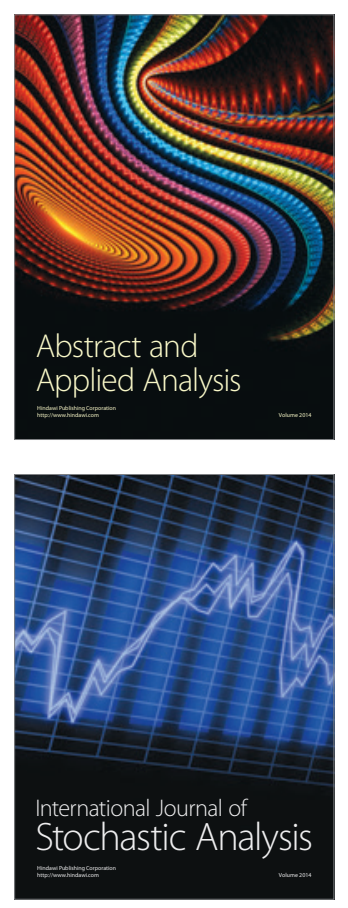

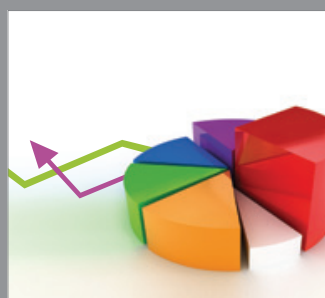

ournal of

Probability and Statistics

Promensencen
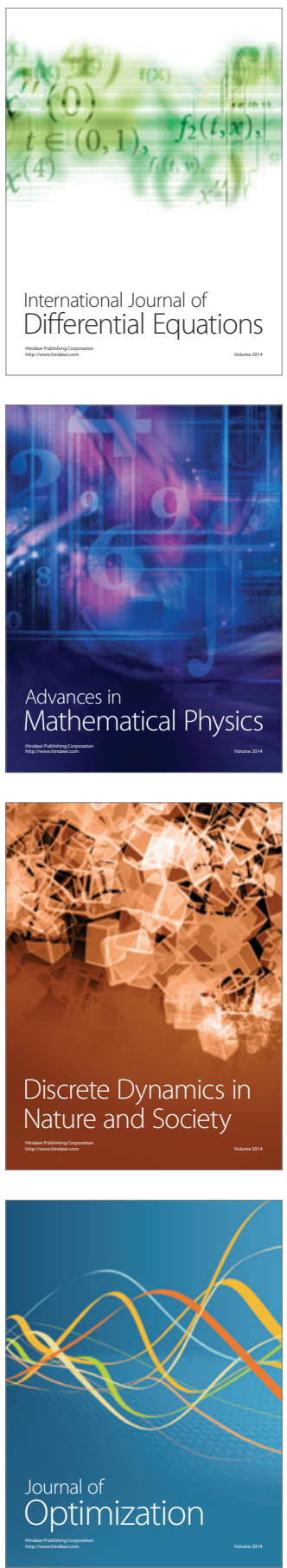